\title{
Stretching the Bounds of 3D Printing with Embedded Textiles
}

\author{
Michael L. Rivera ${ }^{1}$, Melissa Moukperian ${ }^{2}$, Daniel Ashbrook ${ }^{3}$, \\ Jennifer Mankoff ${ }^{1}$, Scott E. Hudson ${ }^{1}$ \\ ${ }^{1}$ Human-Computer Interaction Institute \\ Carnegie Mellon University \\ $\{$ mlrivera, jmankoff, \\ scott.hudson\}@cs.cmu.edu \\ ${ }^{2}$ College of Imaging Arts and Sciences \\ ${ }^{3}$ Golisano College of Computing \\ and Information Sciences \\ Rochester Institute of Technology \\ \{daniel.ashbrook, mdmfaa\}@ rit.edu
}

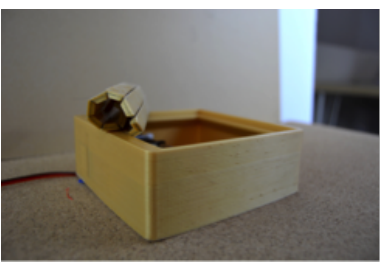

(a)

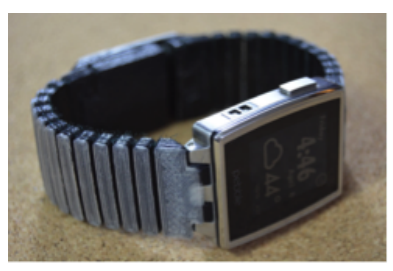

(b)

Figure 1. A range of textile-embedded 3D printed objects fabricated using our techniques - a box with a rolling lid containing a mesh of polyester and strings for actuation (a); a functional watchband printed on a polyester mesh (b); a figure with a pressure-sensitive head that controls an embedded displacement sensor containing a mesh of nylon and spandex fibers (c); a 22 inch $(56 \mathrm{~cm}) \mathrm{crown}$ printed on a single piece of felt larger than the print bed (d).

\section{ABSTRACT}

Textiles are an old and well developed technology that have many desirable characteristics. They can be easily folded, twisted, deformed, or cut; some can be stretched; many are soft. Textiles can maintain their shape when placed under tension and can even be engineered with variable stretching ability. Conversely, 3D printing is a relatively new technology that can precisely produce functional, rigid objects with custom geometry. Combining 3D printing and textiles opens up new opportunities for rapidly creating rigid objects with embedded flexibility as well as soft materials imbued with additional functionality. In this paper, we introduce a suite of techniques for integrating 3D printing with textiles during the printing process, opening up a new design space that takes inspiration from both fields. We demonstrate how the malleability, stretchability and aesthetic qualities of textiles can enhance rigid printed objects, and how textiles can be augmented with functional properties enabled by $3 \mathrm{D}$ printing.

\section{Author Keywords}

Additive manufacturing; 3D printing; soft materials; textiles; interactive devices.

\section{ACM Classification Keywords}

H.5.2. [Information Interfaces and Presentation (e.g., HCI)]: User Interfaces.

\footnotetext{
Permission to make digital or hard copies of all or part of this work for personal or classroom use is granted without fee provided that copies are not made or distributed for profit or commercial advantage and that copies bear this notice and the full citation on the first page. Copyrights for components of this work owned by others than ACM must be honored. Abstracting with credit is permitted. To copy otherwise, or republish, to post on servers or to redistribute to lists, requires prior specific permission and/or a fee. Request permissions from Permissions@acm.org.

CHI 2017, May 06-11, 2017, Denver, CO, USA

(C) 2017 ACM. ISBN 978-1-4503-4655-9/17/05 ...\$15.00

DOI: http://dx.doi.org/10.1145/3025453.3025460
}

\section{INTRODUCTION}

Textiles are a very well developed technology: techniques for the construction, manipulation, and modification of textiles have been refined for millennia [5]. Textiles offer many desirable characteristics: they can be easily folded, twisted or deformed; some can maintain shape when placed under tension and preserve functional qualities when cut. These and other properties such as aesthetic appearance, warmth, and versatility have afforded textiles a rich history of practical uses extending from clothing and decoration to more functional objects such as furniture and even as parts of buildings [6].

In contrast, 3D printing is a relatively new technology that offers a means of fabricating items with precise custom geometries. While most 3D printing techniques create rigid objects-typically from plastic and more recently from metalwe believe 3D printed objects can benefit greatly from the flexibility, stretchability, and aesthetic qualities found in many textiles. Similarly, the utility of textiles can be augmented by the use of computer-aided design software and the accuracy and functional properties of 3D printing. As illustrated in Figure 1, the combination of 3D printing and textiles creates a new design space for rigid objects with embedded flexibility and soft materials imbued with functionality. In this paper, we present the following contributions:

- we introduce a series of design primitives enabled by combining 3D printing and textiles;

- we demonstrate a set of techniques for successfully combining 3D printing and textiles, including details on how to successfully adhere plastic to fabric, how to include fabric in a print, and methods for achieving high-quality results; 


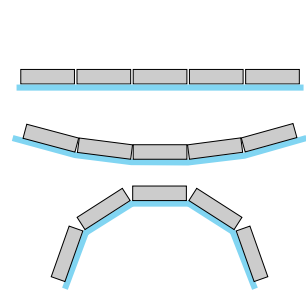

(a)

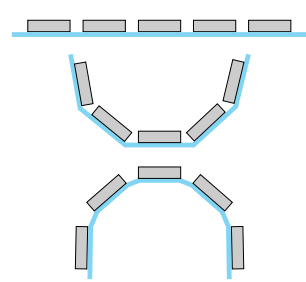

(c)

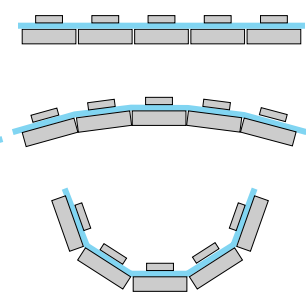

(b)

\section{(d)}

Figure 2. Primitives for stiffening in a single dimension by adding plastic (gray) to fabric (blue). Closely spaced elements printed onto the fabric can prevent it from bending up while allowing it to relax down (a); widely spaced top elements coupled with closely spaced bottom elements enforce the opposite motion (b). Using widely spaced elements allow the fabric to flex in both directions (c), while a solid bar prevents flexing entirely (d).

- we show how embedding textiles can enable the creation of customized input devices on consumer printers in new ways;

- we illustrate how our techniques enable the rapid creation of objects larger than the print bed.

We show each of these techniques and demonstrate their utility through an array of examples, starting with reusable design primitives and mechanisms which can be combined to create useful objects. For example, Figure 1a shows controlled bending being used in a retractable box lid, demonstrating how the direction, degree, and shape of bends can be controlled by fabricating plastic elements on fabric. We also show the benefits of 3D printing for fabric layers that extend beyond the print bed (Figure 1d), creating arbitrarily shaped reinforced holes (grommets) in fabric, and providing one way of addressing the problem of fast prototyping. We show working examples of input devices such as buttons (Figure 1c), sliders, and knobs that take advantage of the stretchy nature of knit fabric to sense user input. In addition to supporting the implementation of basic sensing mechanisms, fabric conveys other benefits. For example, a mechanical slider can make use of the stretchable properties of spandex to re-center its slider thumb.

\section{RELATED WORK}

The properties of textiles have long been explored in the material science and textile manufacturing communities $[13,15$, $14,11]$. Their structure of interlocking fibers-either natural or manufactured-gives textiles a breadth of characteristics that are useful beyond their appearance. Some textiles can absorb sound or moisture, and be engineered with specific stretchability [15]. Others may be coated with polymers that block wind and water, or are sensitive to heat [32].

Fabric - the material produced by interlacing fibers through weaving, knitting, crocheting, or bonding_can also be de-

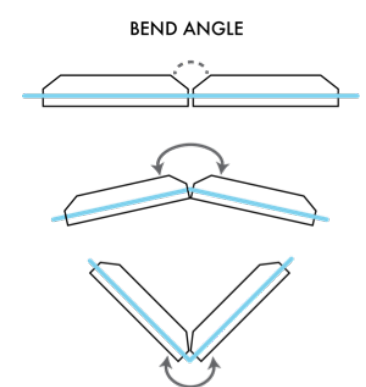

Figure 3. Plastic segments that restrict the bend angles of a textile.

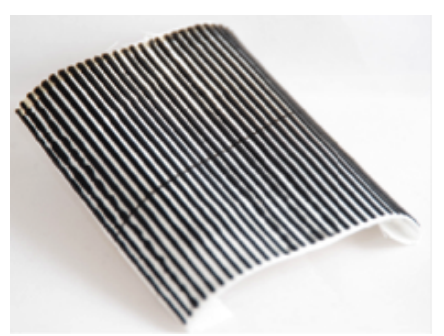

Figure 4. A textile that is only capable of rolling or flexing along the axis parallel to the plastic segments.

signed to provide further variations in properties such as stretchability and permeability. For example, fabric engineered with large spaces between fibers, or interstices, can facilitate faster diffusion of liquids such as water or hot plastic between the fibers [36]. Shaping and/or stiffening of fabric is accomplished by attaching it to rigid objects, draping it over rigid objects, sewing seams and darts, and layering fabrics. These are all fairly labor-intensive actions that require some skill and knowledge about sewing and textile-working.

Textiles have recently captured the imagination of the 3D printing community, which can benefit from aesthetic and other properties of textiles, while reducing the manual labor needed to create with them. For example, Hudson [17] described a new type of 3D printer that prints in needle-felted yarn, enabling the creation of new soft objects such as teddy bears. Peng et al. [27] created a layered fabric printer, which uses textiles as the printing medium, cut with a laser cutter and adhered together using an iron and heat sensitive adhesive. This printer could combine two different textiles, enabling, for example, the embedding of conductive material in a print.

Others have chosen to manipulate the aesthetic properties of 3D printed objects without changing the underlying materials. For example, HapticPrint adds surface textures for primarily aesthetic purposes [35] while Laput et al. show that a consumer fused deposition modeling (FDM) printer can create hair-like extrusions without modification to the hardware [19]. Melnikova et al. explore a variety of lace-like structures and knit-like structures using combinations of hard and flexible materials laid down using either selective laser sintering (SLS) or FDM machines [20].

There has been limited prior research into combining FDM printing with existing textiles. Pei et al. show that it is possible to directly 3D print plastic onto textiles [26], exploring 


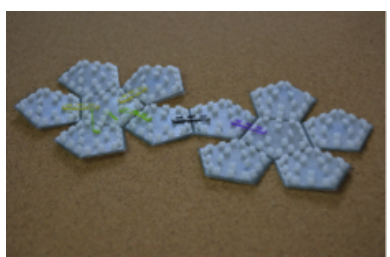

(a)

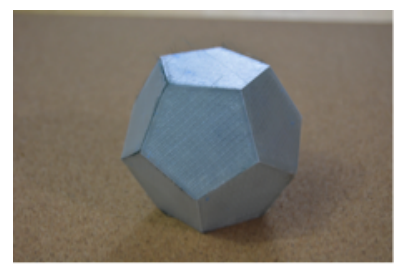

(b)
Figure 5. A shell printed dodecahedron fabricated by printing through a polyester mesh. The faces of the object were printed flat on a single sheet of fabric that was cut post-printing (a). Rubber bands we used to actuate the shell mesh into final 3D for (b).

structures such as a latch and hook, and demonstrating the potential for printing braille or even an articulated part affecting flexibility. They also examine the quality of adhesion acceptability via visual and surface inspection, suggesting PLA has strong adhesion with certain fabrics. However, they do not measure the force that can be held by a bond between plastic and fabric nor the extent to which a fabric will stretch before the bond breaks. Mikkonen et al. create flexible, 3D printed objects intended to be integrated with cloth [21]. However, they use standard methods for attaching hard objects to textiles, such as printing buttons and sewing them on, or inserting boning into a corset. Sabantina et al. demonstrate simple 3D printed forms combined with textile structures and their mechanical and geometric properties [31]. Our work extends theirs by realizing a set of usable design primitives, techniques and exemplars that support combining textiles with 3D printed components, and provides direction and guidance for exploring this design space. Finally, 3D printer manufacturer 3D Systems added fashion-oriented printing-onto-fabric capabilities to its consumer-level Cube printer shortly before discontinuing production of the printer [1].

In the domain of textiles, and particularly fashion design, the ability to add structure and manipulability to fabric has long been of interest, although less of the focus has been on fabrication methods, and more on fabrication outcomes. For example, computer-aided design (CAD) and manufacturing (CAM) have long been an aspect of fashion design. Driven in part by the needs of realism in animation, textiles can now be modeled in great detail $[4,7]$.

Augmentation of textiles in various ways has also long been a focus of interest, from Spyn's approach to attaching storytelling to knitting [30] to TeeBoard's ability to support easy embedding of electronics in clothing [24]. Combining technology and textiles has become more accessible to end users (e.g., $[28,29])$ and is a domain in which innovation takes place as much by hobbyists as by academics [2]. For example, a recent Instructable describes some basic techniques for directly printing onto fabric [18]. Our work expands the set of techniques for successful printing (e.g., discussing how to compensate for issues like tilting and sagging of fabric during printing, selective adherence control, and combinations needed to fabricate devices like a retracting mechanical slider).

Another area of relevance is the shaping and mechanical articulation of 3D objects (see [12] for a survey of this area). Here we may draw from origami [10], marionette design [8],

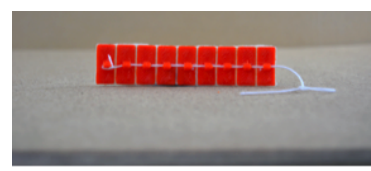

(a)

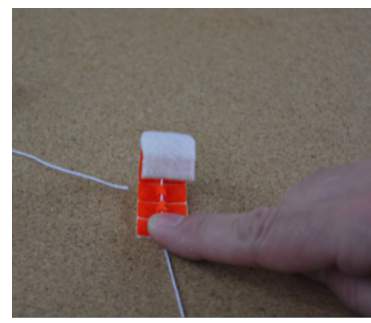

(c)

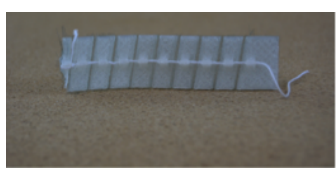

(b)

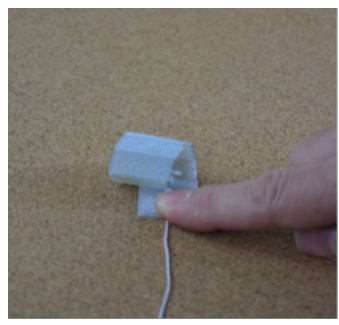

(d)
Figure 6. Textile-embedded, string actuated mechanical arms with variable bend angles- (a) and (c) show straight segments of plastic lead to a simple roll that aligns with the axis of the channel; (b) and (d) show angled segments of plastic cause the roll to skew to the left.

materials engineering [33], mechanism design [9], and shapechanging interfaces $[37,16,25]$. However, our work expands this design space by accommodating a different set of tradeoffs due to different materials and fabrication techniques.

\section{DESIGN PRIMITIVES}

Our exploration of textile-embedded printing takes place at three different levels: fully working objects (we discuss six examples at the end of the paper); low-level techniques for successfully 3D printing with textiles; and design primitives for accomplishing specific higher level goals. In this section, we introduce these design primitives, showing how the differing properties of textiles and plastic can complement each other. In subsequent sections, we explain techniques for achieving this fusion of materials, and illustrate a number of fully-working examples.

\section{Selective Stiffening}

Adding plastic to fabric enables selective stiffening of the fabric, controlling where and how the fabric can bend or stretch. Applying this basic primitive allows us to build up a number of higher-level primitives, including mechanically-actuated devices, interactive, sensor-based objects, and larger scale fabrications using fabric skins.

Printed plastic normally cannot bend unless hinges are included in its design, which often requires a level of precision hard to achieve in consumer grade printers and/or additional assembly steps. In contrast, most common textiles are not normally rigid at all. Many techniques exist for adding stiffness to fabric, such as incorporating additional hard materials (e.g., cardboard or plastic whalebone); however, adding these materials can be labor-intensive and require considerable knowledge of and skill in sewing and textile shaping.

By printing plastic onto fabric, we can selectively stiffen different areas of the fabric, controlling how the fabric is able to bend (Figure 4). Figure 2 illustrates the different ways we can control bending in a single dimension. By controlling the spacing of $3 \mathrm{D}$ printed elements on top of fabric, we can allow or prevent bending in the direction perpendicular to the fabric. 


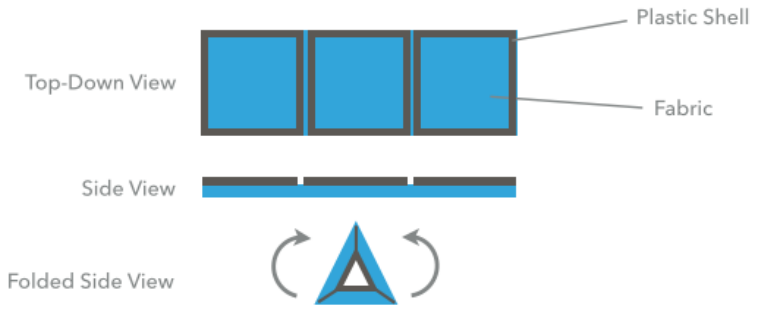

Figure 7. A shell printed triangular prism first printed flat and then folded to its 3D form.

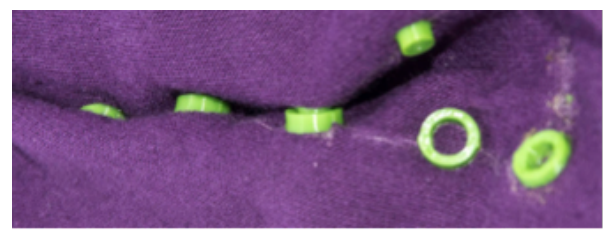

Figure 8. 3D Printed snaps printed onto a fabric.

Incorporating the fabric mid-print allows us to control bending in the opposite direction as well.

In addition to controlling direction of bend, we can also control degree of bend by manipulating the amount and shape of added plastic. Specifically, the bend angle can be controlled by using closely spaced stiffeners with angled side cutoffs or explicit side braces. As illustrated in Figure 3, the bend angle will be approximately the sum of the two side angles in the bend direction, and will remain small in the opposite direction (unless additional bending support on the opposite side is provided). Note however, that the exact bend angle may depend on the stretch of the small section of fabric between the stiffeners. Consequently, for fabrics with very high stretch it may be necessary to experiment to obtain an exact angle.

Individual one-dimensional bends can be composed to create more complex shapes and motions. For example, Figure 6 illustrates curling shapes produced using angled and straight stiffening segments. Figure 5 illustrates the creation of a 3D solid shape by folding from a flat object. We achieve the final shape by printing each edge in the polygonal model with a pair of braces that have an bend angle equal to half the dihedral angle for that edge.

\section{Selective Adherence}

By selectively adhering plastic to the fabric, we can control the degree of flexing and stretching in the plane of the fabric, in addition to bending out of it. We can further customize behavior by selectively manipulating the areas where plastic adheres to the fabric. We can control adherence by placing painter's tape over areas of the textile where plastic is placed but should not adhere. We are then free to print on and around the tape, which supports the hot plastic while preventing it from bonding with the surface below. After printing is complete, the fabric can shift or stretch while being in contact with, but not adhered to, the plastic. This technique allows us, for example, to create a mechanical slider which is free to move and retract using stretchy fabric (Figure 18b).

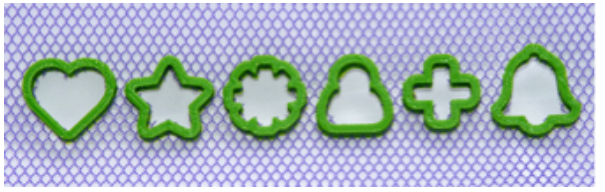

Figure 9. Arbitrarily shaped grommets 3D printed onto a polyester mesh. The centers of the grommets were cut out after printing.

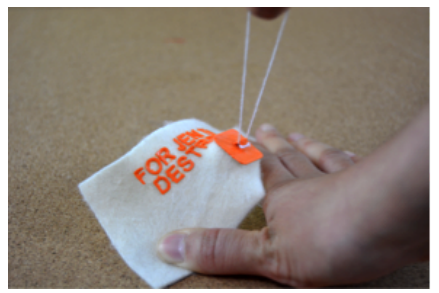

Figure 10. Plastic extruded onto a textile with a fuzzy surface creates very strong adherence. The plastic seen here is difficult to separate from a piece of felt.

\section{Mechanical Action}

By building on the stiffening primitives, we can begin to create more complex interactive devices. Constraining the bend of the fabric in specific ways can create simple mechanisms. Figures $6 \mathrm{a}$ and $6 \mathrm{c}$ illustrate how a string affixed to one end of a strip of stiffening segments can create a rolling mechanism. By varying the angle at which the segments are placed next to each other, the strip can be made to curl as it rolls up (Figures 6b and $6 \mathrm{~d}$ ). Figure 1a illustrates a hybrid textile/printed box with a segmented lid. Controlled by a motor, the box's lid can automatically curl open and uncurl closed.

\section{Fast Production}

Current FDM printing is slow, taking many hours to fabricate larger objects. Previous research has explored different methods to speed up the printing process such as printing in lower fidelity [22, 23] or reusing previously printed objects [34]. However, fabricating plastic layer-by-layer is still inherently time-consuming.

One of the key advantages of using textiles with 3D printing is that layers of fabric are pre-fabricated, opening up the possibility of saving print time by selectively replacing some of the printed plastic with fabric. However, to emulate the hard plastic it replaces, the fabric may need to be reinforced so that does not bend. We may stiffen fabric with a small amount of plastic support printed over the surface, similar to use of wireframes for faster printing by Mueller et al. [22]. We term the technique for fashioning objects like this "shell printing" because we construct the outer shell of an object using textile panels created with this method (Figure 7).

There are several options for assembling a shell print. The easiest methods are to add structure for fastening shells together such as 3D printed snaps (Figure 8) and clips, glue the panels together at the edges. If desired, the panels can also be sewn.

\section{Large Objects}

An advantage of printing on fabric is that it can be larger than the print bed, allowing a print to be "continued" in 2D. This allows us to repeatedly print on the same piece of fabric, 


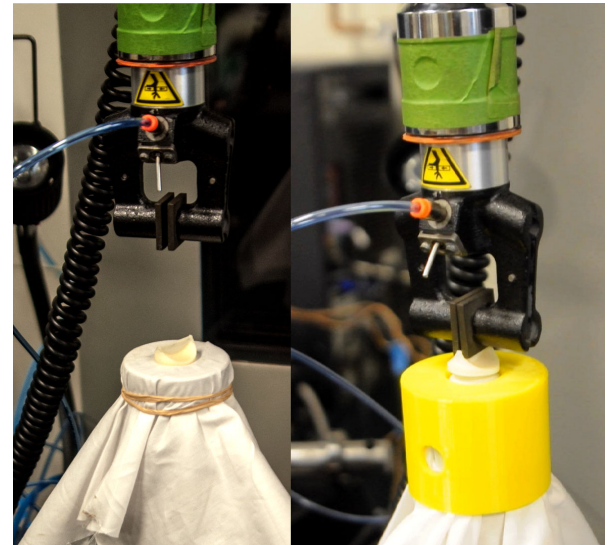

Figure 11. Instron adhesion test setup. Left: attachment of fabric and plastic sample to test column; right: fabric anchor (yellow) with test in progress.

repositioning it on the print bed and moving already printed sections out of the way, resulting in a continuous print larger than the printer itself. The restriction of this approach, similar to other work involving adding to existing fabrication [34], is that to prevent obstructing the extruder's movement, the gaps between each printed section must be larger than the size of the extruder nozzle. Another issue that arises is proper alignment of a textile such that printing can be done where desired. One solution is to use a skirt layer to assist in positioning the textile on the print bed. We have also successfully experimented with using a laser pointer attached to the extruder head to guide alignment of a print each time the textile is moved. Figure 1d illustrates a wearable object longer than the print bed fabricated in this way.

\section{Reconfiguring Textiles}

Textiles have many options for post processing. For example, assembly of a shell print can be done through printed snaps (Figure 8) or textile-working techniques like sewing. A textile can be cut to size or shape after printing to remove excess material; it can also be cut to adjust a part if the exact sizing is not known at design time. Reinforcing plastic may be printed around holes - creating grommets - to protect the fabric's raw edges from fraying and provide passage for cords or strings. Figure 9 shows several examples of arbitrary grommet shapes.

We note that augmentations such as snaps and grommets can be readily provided by more conventional means. However, having a way to $3 \mathrm{D}$ print these as well allows us to reduce the number of separate manufacturing steps to create an object. In addition, it provides a flexible way to mix these conventional fixed form augmentations with the kinds of highly custom structures which are most readily achieved with $3 \mathrm{D}$ printing.

\section{Summary}

The addition of fabric in the context of consumer-grade 3D printing enables a set of novel design primitives that printed plastic alone cannot realize. With stiffening constructs, we can control how the fabric flexes; composing these allows for more complex flexing behavior and allows for mechanical properties such as rolling and snapping. Using a material such as painter's tape, we can selectively prevent the plastic from adhering to the fabric, allowing for further movement possibilities.

\section{BASIC TECHNIQUES FOR 3D PRINTING WITH TEXTILES}

Strong adherence between textiles and 3D printed plastic is necessary to achieve the properties we describe above. We conducted extensive experiments - both informal and formalto determine the best methods to achieve a strong bond, as well as techniques for combining our design primitives to create more complex structures.

In our experiments, we used unmodified consumer-grade FDM 3D printers, PLA and ABS filament, and a variety of different textiles. Note that there is inherent variability in measurements related to our process. The temperature for $3 \mathrm{D}$ printing a plastic varies based on the calibration of the $3 \mathrm{D}$ printer as well as the quality and type of filament used [3]. For example, the ideal print temperature of PLA is typically between 180$230^{\circ} \mathrm{C}$. Similarly, coatings and variations in material makeup of fibers may alter how a textile interacts with printed plastic. Information about the presence and types of coatings on textiles is not easily available, particularly if textiles are purchased off the shelf. Thus some degree of experimentation around parameters such as extrusion temperature is required when attempting to print with embedded textiles.

The two main factors we consider for successfully combining fabric and plastic are adherence and stability. Adherence refers to the ability of the plastic filament to attach to the textile, while stabilizing the fabric with respect to the printer is also necessary to achieve success, particularly when printing tall objects.

\section{Adherence}

Because FDM printing lays down print material in layers, it is critical that each layer bonds to the previous one. When incorporating textiles in an FDM print, the textile can be considered a "layer" as well.

Due to variability in factors such as printer temperature and textile coatings, experimentation is required to attain optimal adherence, such that the textile cannot not be easily separated from a print (Figure 10). We found that PLA, for example, often adheres better to fabric when extruded at a slightly higher temperature than typical $\left(210-220^{\circ} \mathrm{C}\right)$. The high temperature produces lower viscosity and facilitates a longer settling period for the plastic to cool and solidify, allowing it to partially seep into the fabric for a stronger bond.

For thin fabric, approximately less than $0.4 \mathrm{~mm}$ thick, the best adherence resulted from simply securing the fabric and proceeding with printing without leaving extra space in the model for the fabric layer: the force and heat of the extruder pressing into the fabric assisted in achieving a good bond between the plastic and textile. For thicker fabric, however, the extruder can catch on the fabric, displacing it during the print process. In this case, it is necessary to raise the height of the next layer of plastic to be printed, leaving space for the fabric.

When a fabric is not used as the first layer of a print but instead sandwiched the fabric between two layers of plastic, it will not 


\begin{tabular}{clclll} 
ID & Name & Treatment & Composition & Thread count & Weight \\
\hline A & Ripstop Nylon & - & $100 \%$ nylon & 196 & 68 \\
B & Sport Nylon & - & $100 \%$ nylon & 113 & 91 \\
C & Symphony Broadcloth & - & 65\% polyester, 35\% cotton & 208 & 112 \\
D & Voile & - & 65\% polyester, 35\% cotton & 120 & 53 \\
E & Premium Muslin & Bleached & $100 \%$ cotton & 204 & 125 \\
F & Sew Essential & Bleached & $100 \%$ cotton & 142 & 114
\end{tabular}

Table 1. Test fabric properties. All fabrics tested were plain weave. Thread count is threads per square inch and weight is grams per square meter.

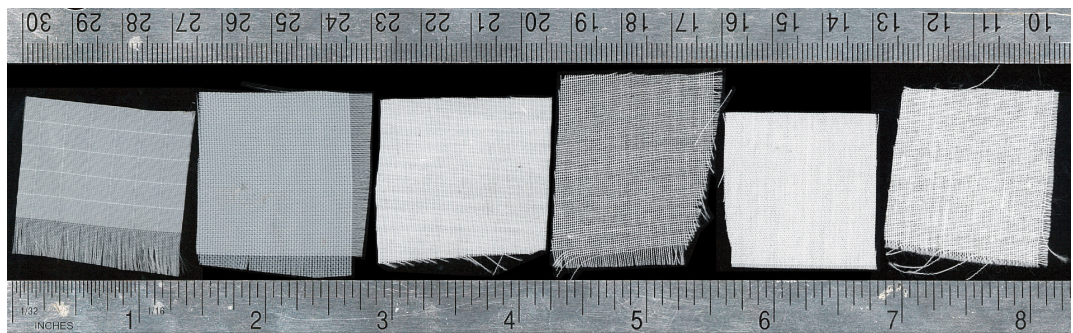

Figure 12. The fabrics used in strain testing; in order of Table 1 (A-F) from left to right. The top ruler is in centimeters; the bottom is in inches.

normally adhere to the layer below it. If it should be adhered, the fabric must have holes, or interstices, for the melted plastic to flow through. These openings may be an intrinsic part of the fabric, in the case of netting or other low-density textiles, or can be artificially created by cutting.

\section{Adhesion Testing}

Pei et al. examine the quality of adhesion between plastic and fabric through visual and surface inspection [26]. However, they do not provide quantitative insights about the bond strength. We achieved a preliminary characterization of the bonding performance of plastic and fabric using an Instron model 5567 strain tester to measure the force required to separate plastic printed onto different types of fabric. Figure 11 illustrates our testing setup: we printed a 1-in $(2.54 \mathrm{~cm})$ diameter "plug" of plastic on a sample of fabric, affixed it to the lower arm of the Instron and attached the upper arm's clamp to the plug. The machine then pulled the two arms apart until the plug separated from the fabric, measuring the amount of force required during the extension.

We measured a baseline of performance using basic materials and default printer configurations. We performed the test using two plastic types, ABS and PLA, both un-colored, from Mitus ${ }^{1}$. Our fabric samples were similarly un-dyed and, as much as we were able to determine, un-coated. We printed the plastic at manufacturer-recommended temperatures $\left(248^{\circ} \mathrm{C}\right.$ for $\mathrm{ABS}$ and $210^{\circ} \mathrm{C}$ for PLA) on an Ultimaker 3D printer. Figure 13 illustrates the results of our adherence test. Table 1 describes the properties of the six fabrics we tested, and Figure 12 shows images of each fabric.

Our results demonstrate that the adhesion capabilities can vary widely based on the composition of a fabric and the filament used for printing, which is useful in supporting different functionality. For example, the combination of Sew Essential fabric with PLA is capable of sustaining a weight of $50 \mathrm{~N}$ (11 pounds of force) which is equivalent to a grocery bag full of food. Similarly, the Voile fabric with PLA can extend about $1 \mathrm{~cm}$ in length with a small amount of force (approximately

\footnotetext{
${ }_{1}^{1}$ http: //mitusbrand. com
}

$10 \mathrm{~N}$ or 2 pounds of force) without adhesion failing. This property can be used to support mechanical action such as retraction in a slider.

\section{Stabilization}

When $3 \mathrm{D}$ printing with plastic alone, as long as the printer is calibrated and the bed is appropriately prepared, the plastic will adhere, providing a stable surface for further layers of printing. Unfortunately, the mechanical advantages of fabricstheir abilities to compress, flex, and stretch-are also potential disadvantages when it comes to the actual print process. For example, the relative motion between the print head and the fabric can cause lateral forces to be exerted on the fabric which can then shift or stretch; fabric placed across gaps can sag; and taller prints can tilt or twist as the fabric flexes. Extra steps that prevent these issues must be taken to provide a stable surface on which to print. We describe solutions below and illustrate them in Figure 14.

\section{Shifting}

Unless a piece of fabric is secured to the print bed, it will simply be pushed around by the print head as it moves. As discussed in the Section Adherence, for thicker fabrics we can position the print head such that it does not catch, but this can lessen adherence. Textiles with a low degree of stretch may be fixed in place at the edges with tape or clamps similar to [18]. For fabric with a higher degree of stretch, it may be necessary to secure the underside as well to prevent shifting during the print process. In our experiments, we found that double-sided tape was effective at preventing this unwanted motion.

For fabric that is intended as a middle layer of the print-for example, in our displacement sensor in Figure 15-the same technique of fixing the fabric in place at the edges applies. Alternatively, if it is undesirable to drape the fabric over the edges of the print, another strategy is to use glue. In our experiments, we found that gel-viscosity cyanoacrylate adhesive was highly effective in bonding fabric pieces mid-print. 


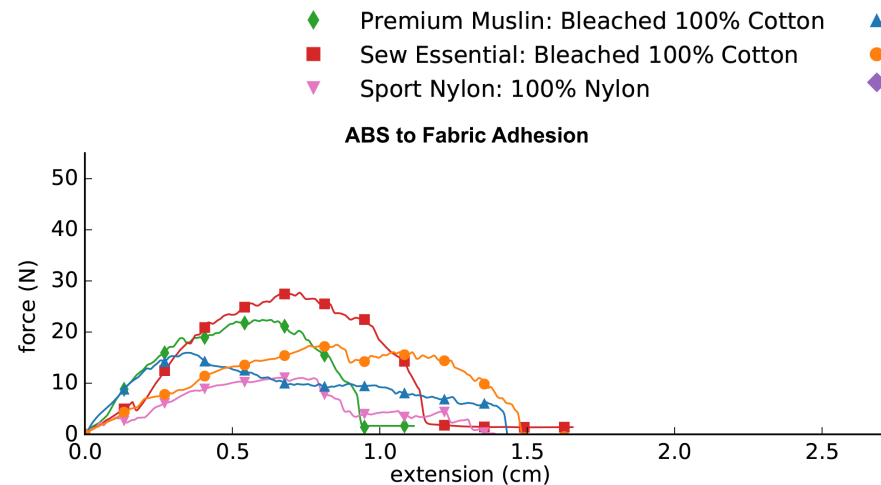

Symphony Broadcloth: $65 \%$ Polyester 35\% Cotton
Voile: $65 \%$ Polyester 35\% Cotton
Ripstop Nylon: $100 \%$ Nylon

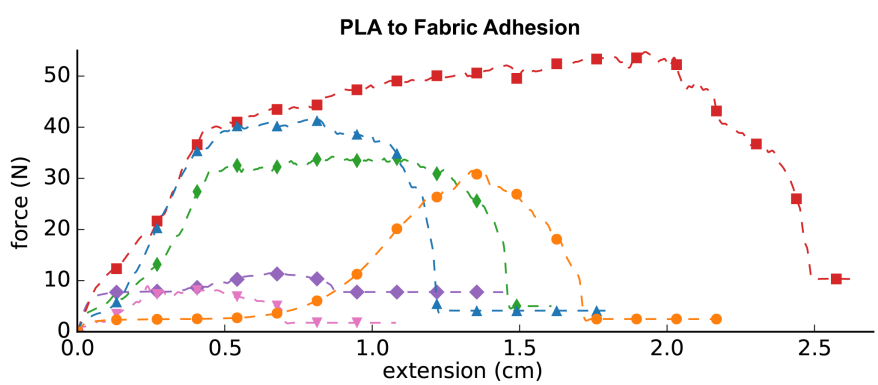

Figure 13. Results of adherence testing showing force exerted for a given extension. Both graphs are on the same scale; the left is for ABS and the right is for PLA.
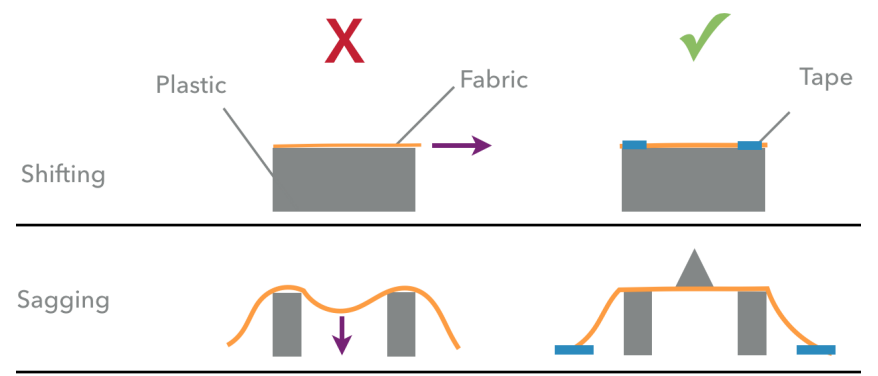

Tilting

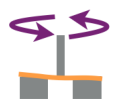

Figure 14. Stabilization problems and solutions.

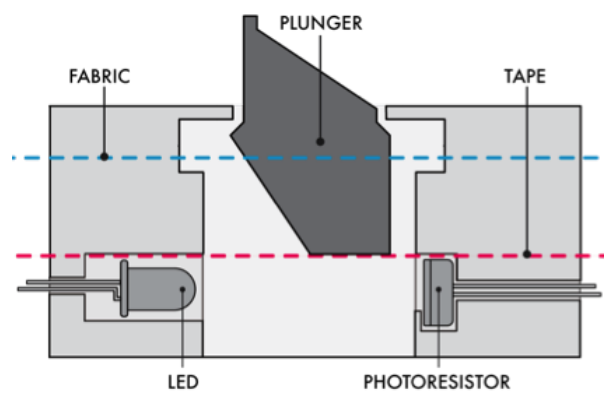

Figure 15. Cross-section of a textile-embedded displacement sensor. The tape is inserted with electrical components during the printing process. The tape acts as temporary support for printing the plunger. It is later removed using an opening on the underside of the object (Figure 16).

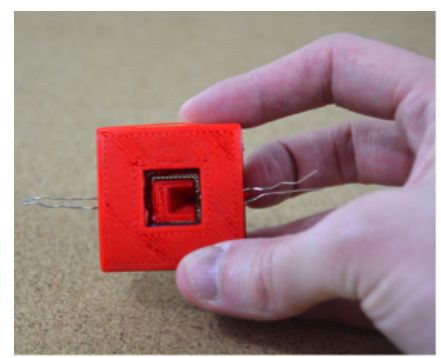

Figure 16. The underside of the displacement sensor showing the suspended plunger.

\section{Stretching}

The ability of some textiles to stretch adds opportunities to be embedded into 3D prints. For example, stretch can facilitate the creation of resizable objects and even pressure-sensitive input devices (Figure 1c). However, stretch can also create problems by introducing movement that makes objects tilt as more layers added to them. Additionally, textiles made of nylon or spandex fibers may shift or melt due to contact with the extruder. Calibrating the extruder to print just above the surface of the textile and placing painter's tape over it can reduce stretching as demonstrated by [18].

\section{Sagging}

An advantage of textiles is their ability to easily bridge large gaps in a print. However, they may sag if not well supported and printing may cause the fabric to dip, leading to improper layer alignment and poor adhesion of plastic. This problem can be reduced by first placing painter's tape over a gap to support the fabric (Figure 17c), or by using one of the shiftingreduction methods to keep the textile taut over the gap.

\section{Tilting}

Tilting occurs when the object being printed has a small contact area with a textile and a comparatively large height. As the height rises, it is more likely that the print head will be able to push the object aside causing inaccurate (or even in-air) printing. This effect is worsened by textiles that stretch. We can correct tilting by placing painter's tape directly on a textile near problem spots to reduce stretching, or by adding doublesided tape between the textile and the print bed or underlying printed support material to hold it steady.

\section{Printing Beyond a Single Layer}

A fundamental limitation of printing on fabric is that the fabric must lie horizontal to the print plane. Otherwise, it may obstruct the movement of the extruder and prevent hot plastic from being properly laid down. Other research has investigated layering fabric [27], but this technique - in addition to requiring a custom 3D printer and pre-loaded fabric-constrains printing to only textile layers and does not incorporate plastic. Our approach does not have these constraints.

Textiles intended to fold may be printed flat and then folded (such as those described in the Section Design Primitives). Fabric may also be folded up and around after some number of plastic layers are printed, by pausing the printing process to 


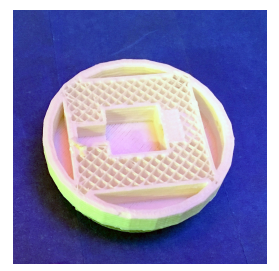

(a)

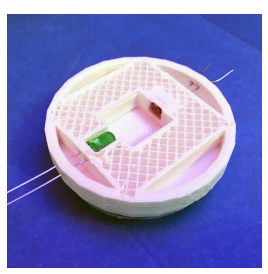

(b)

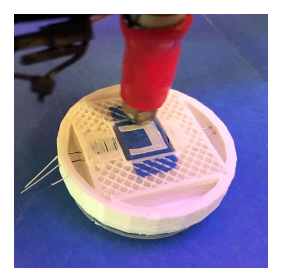

(c)

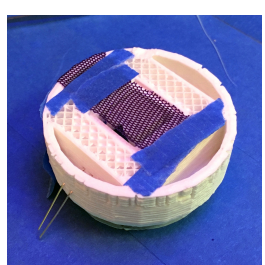

(d)

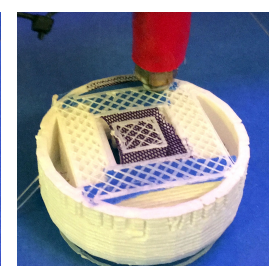

(e)

Figure 17. Process for a fabricated pressure sensitive button. The print is paused when it has enough layers to house the electrical components (a). The electronics are inserted (b). A piece of painter's tape is inserted to bridge the gap between the electronics to support printing the plunger (c). The print is paused again after enough layers have been printed for the plunger so that a stretchable fabric can be laid across the gap and affixed using painter's tape (d). The printing process is resumed and the subsequent layers of plastic adhere to the fabric and the plastic below (e).

place the textile. In many cases, excess fabric is small enough to fit on the print bed, and naturally remains out of the way as layers are added. In other cases, fabric can be folded or rolled and secured out of the way of any moving parts during printing. For our largest print, a felt crown measuring 22 inches (56 $\mathrm{cm}$ ) in length, we were able to fold the felt such that only one segment of the crown was on the print bed at any given time. The remaining fabric rested to the side in a direction that did not interfere with the extruder or print bed motion.

\section{Summary}

Textiles are easy to incorporate into the 3D printing process with unmodified consumer-grade technology. Although any given design requires some experimentation to be successful, such experimentation is common with non-fabric based prints as well. We are confident that others can easily adopt our techniques to incorporate textiles into their 3D prints.

\section{EXAMPLES}

In this section, we present a series of ten end-to-end examples that demonstrate applications of our design primitives to create novel printed objects, ranging from interactive devices to wearables and toys.

\section{Input Devices}

Our design primitives can be used with stretchable textiles to quickly fabricate customized input devices. We created a reusable displacement sensor whose electrical components are embedded during the printing process, as illustrated in Figure 15. The displacement sensor allows custom actuation mechanisms to be quickly fabricated, snapped on top, and tried out before deciding on a final design.

The principle of operation behind the displacement sensor (Figure 18a) is to sense the movement of a fabric-suspended plunger: when the plunger is moved, it occludes light produced by an LED in proportion to the amount of displacement. We detect this change using a photoresistor placed directly across from the LED inside of the object.

The inside of the displacement sensor is hollow to allow the the plunger to move freely (Figure 15). During the printing process, we temporarily support the plunger with tape (Figure 17c). Once enough layers of the plunger are printed, we pause the print and securely lay down a thin stretch fabric composed of nylon and spandex fibers (Figure 17d). We resume the print to finish fabrication of the object (Figure 17e), embedding the fabric partially inside the plunger. Finally, we remove the support tape through the opening on the underside of the object (Figure 16). This leaves the plunger suspended in mid-air by the stretch fabric.

Once the base of the displacement sensor is constructed, we may snap on additional actuation mechanisms to test. Here we illustrate a slider, knob, button, and a final custom shape.

Slider: The slider thumb is constructed using a stretchable polyester mesh, which causes it to snap back to its position in the middle of the box shown in Figure 18b when released. As shown in Figure 15, the displacement sensor is wedge shaped. This causes its height to vary linearly as the slider thumb moves across it.

Knob: We convert rotation into pressure on the displacement sensor using a 3D printed threaded screw with a knob printed on top (Figure 18c). As the knob is turned, it gradually lowers to displace the plunger. The displacement of the plunger is proportional to the amount of rotation.

Button: The pressure-sensing button applies mechanical force to the top of the plunger (Figure 18d). Tension in the textile under the displacement sensor opposes downward motion while allowing the textile to slowly stretch. Once the pressure applied to the plunger is relieved, the mesh retracts and the plunger (and button) return to their initial positions.

Custom Button: As a final example, we demonstrate a custom 3D shape designed around a pressure sensitive button. Using the design primitives of selective stiffening, selective adherence, and mechanical action, we quickly fabricated the inexpensive, "android" illustrated in Figure 1c. Fabric embedded in the android's body supports sensing while giving its head the aesthetic qualities of a bobble-head figure.

\section{Actuated Box with Interactive Control}

Textiles can facilitate the creation of functional objects that have variable stiffness and actuation properties. We created a box with a textile-embedded lid that rolls open upon actuation. The lid was printed with a flexible mesh made of polyester fibers and two strings fixed at one end of the lid for actuation (Figure 19). The button uses the fabric-embedded displacement sensor described in the previous section (Figure 18a).

The lid combines several design primitives. Plastic sections placed along the lid's diagonals demonstrate controlled bending and mechanical action via selective stiffening, resulting in restricting the lid to rolling. The strings are placed through channels on top and bottom of the mesh and are fixed to one 


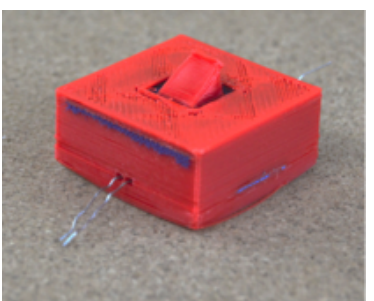

(a)

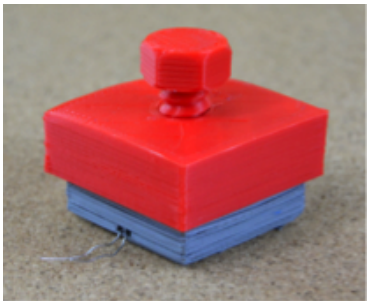

(c)

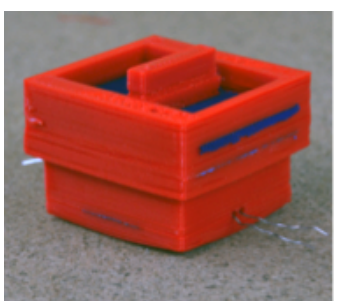

(b)

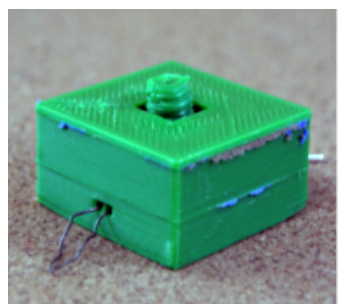

(d)
Figure 18. Textile-embedded input devices- our displacement sensor (a); a retractable slider (b); a knob (c); and a pressure sensitive button (d).

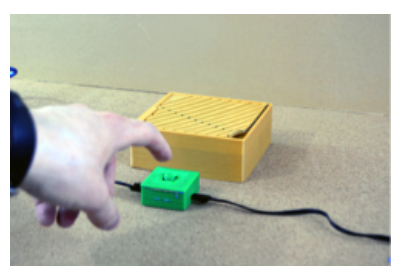

(a)

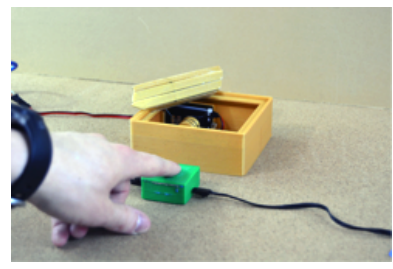

(b)
Figure 19. Textile-embedded box fabricated via controlled bending. The box lid is composed of a polyester mesh and embedded strings that are printed on to allow the box's lid to roll open and unroll closed.

end of the lid such that pulling one results in rolling and pulling the other results in unrolling.

\section{Six-Panel Fabric Lampshade}

Our six-inch (152 mm) cube-shaped lampshade (Figure 20a) was constructed out of five identical panels with a sixth panel modified to accommodate the light bulb (Figure 20b). Each panel was printed separately with short tabs to allow it to be glued to its neighboring panel.

The lampshade demonstrates shell printing. The light bulb mounting point is a type of custom grommet. In addition, the lampshade illustrates reconfiguring via trimming and attachment. While we chose to glue the panels together, other options could include a cord through grommets (e.g., Figure 9) or press snaps (e.g., Figure 8) for easy access to the light bulb. Printing each panel took about 30 minutes and overall the lampshade took three hours from start to full assembly. Printing a full $6 \times 6 \times 6$ inch plastic cube on our printer, in contrast, would take an order of magnitude longer (12 hours for a cube frame or 29 hours for a closed hollow cube).

\section{Folding Polyhedron}

Figure 5 illustrates a dodecahedron designed as a seven-inch $(180 \mathrm{~mm})$ flat sheet of pentagonal faces similar to how the shape would be created via origami. In its folded form, the shape measures three inches $(76 \mathrm{~mm})$ in height. The dodec-

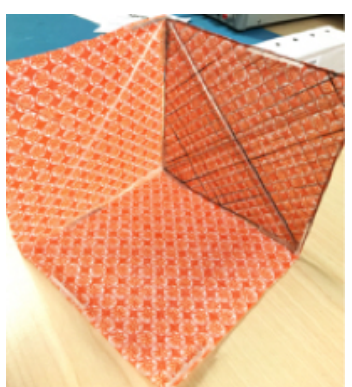

(a)

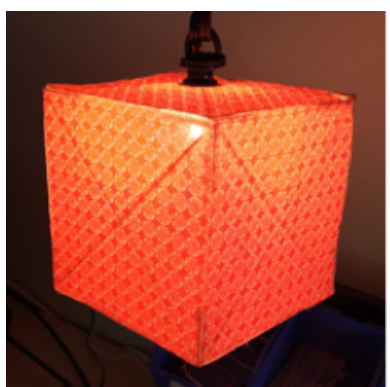

(b)
Figure 20. The shell printed lampshade was constructed by gluing together six panels printed onto fabric (a). The top panel was cut to accommodate a light bulb (b).

ahedron is secured in its assembled position by small rubber bands wrapped around the pegs visible in Figure 5a.

The dodecahedron demonstrates controlled bending using the technique shown at the bottom of Figure 3. A small gap was left between adjacent sides of the shape to create hinges and plastic was used to limit their bending to be close to the proper dihedral angle for a pentagonal dodecahedron $\left(116.56^{\circ}\right)$.

When fabricating the object, we first printed a layer of plastic for the rigid walls of the dodecahedron. A low-density, flexible nylon fabric was then placed above the surface layer and fixed to the print bed. Printing was resumed and the subsequent layers of plastic printed through the fabric adhering to the plastic beneath.

\section{Crown}

We created a wearable, 22 inch $(56 \mathrm{~cm})$ crown, shown in Figures $1 \mathrm{~d}$ and 21 . The crown's decorations were printed as series of rectangular sections onto a single large piece of felt that extended beyond print bed.

We preprocessed the 3D model of the crown to separate it into sections. During the printing process, we aligned each section using a laser pointer attached to the print head, completed its printing, and then readjusted the portion of felt on the bed to print the next section. The crown made use of decorative elements, grommets, and a post-processing step of cutting the felt to the outer shape of the plastic.

\section{Flex Watchband}

The flexible watchband shown in Figure 22 consists of four types of plastic, two layers of fabric, and a magnetic clasp. It is primarily constructed of layers of NinjaTek SemiFlex filament and two layers of polyester mesh fabric. The fabric is folded up and around at the end of the watchband to strengthen the connection around the metal pin that secures the watchband to the watch.

We constructed the watchband from bars of SemiFlex, with only fabric crossing the gaps between them (leading to controlled bending in the direction around the wrist). The fabric is folded up and around the semi-flex to connect the two layers of fabric and provide extra strength.

The watchband went through several design iterations. Early iterations did not prove mechanically robust and lasted only 


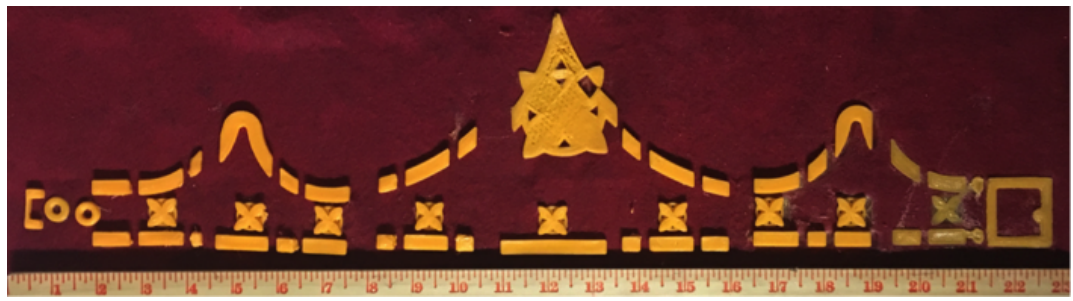

Figure 21. A 22 inch crown fabricated on a single sheet of felt by printing in rectangular sections.
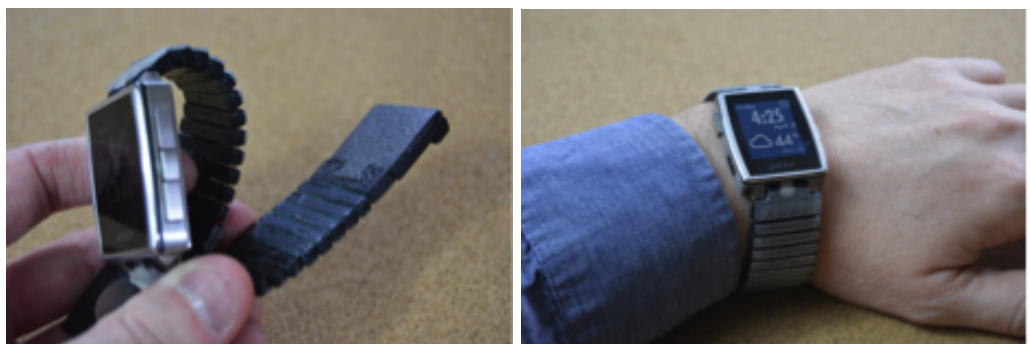

Figure 22. A watchband that supports hinging to a watch face. Stiffeners on the mesh fabric afford flexibility similar to a linked watchband.

a few days before fractures in the brittle material near the attachment pins occurred. We improved robustness where the band connects to the watch-an area with less than $2 \mathrm{~mm}$ of material between the metal mounting pin and the watch bodyby looping fabric around the end of the band, including the metal mounting pins inside the plastic of the band. The fibers of this polyester mesh have higher tensile strength than the printed plastic that they wrap around, and hence substantially strengthen it. The compliant nature of the SemiFlex plastic used for this part of the band eliminates most of the brittleness that would occur with a hard plastic such as PLA.

We used PLA to provide complementary capabilities: this harder material was required for some of the functional parts of the magnetic latch. In addition, we used three separate colors of PLA for aesthetic purposes: translucent to give an overall silver tone to the band, and black and white for a logo on the latch.

After the design improvements outlined above, the band has proven much more robust. It was used daily by one of the authors for about four months (until the watch it was built for began to fail).

\section{LIMITATIONS}

Our examples demonstrate the range of possibilities enabled by combining 3D printing and textiles. However this combination also poses some limitations. Some textiles can experience permanent stretching over time. In our custom button, this effect gave the button a "squishier" feel. Our watchband also stretched slightly over the months it had been in use; choosing a different mesh for the interior of future bands may mitigate this issue.

The durability of textiles embedded in 3D printed objects can be limited as well. Towards the end of its testing period, our watchband experienced some fraying of the connecting fabric near non-flexible latch components; an extra reinforcing fabric layer in these problem spots would likely mitigate this issue. Similarly, we discovered durability is an issue for the strings in the actuated box due to them rubbing each time the lid rolls and unrolls. We used braided gel-spun ultra-high molecular weight polyethylene fishing line to address this issue.

Lastly, for printing objects larger than the print bed, a limitation of our approach is the need to leave large (print-head wide) gaps between sections. Without sufficiently sized gaps, printing artifacts may be produced such as those visible in the rightmost side of the crown in Figure 21. Better software support for dividing up an object and printing appropriate fiduciary markers would help with fabrication and reduce printing artifacts.

\section{CONCLUSION}

We have demonstrated a capability to control, manipulate and mix fabric with $3 \mathrm{D}$ printing. These techniques open a design space that creates new possibilities for rapidly fabricating rigid objects with embedded flexibility and soft materials imbued with functionality.

While we showcase the use of fabric with 3D printing, these techniques are not mutually exclusive with others: different techniques afford different pro and cons. For the watchband, we used a flexible plastic (SemiFlex) because it was soft against the skin and reduced moisture absorption (e.g., from sweat) while fabric could have retained moisture. Similarly, we used fishing line for strings within the actuated box to address durability issues.

Taken as a group, our final examples illustrate the range of new physical objects that are enabled by the use of textiles during $3 \mathrm{D}$ printing. Our techniques create objects that can meet a wider range of human needs by encompassing more pleasing materials and larger sizes than typical consumer-grade 3D prints. In addition, the ability to easily enhance arbitrarily shaped physical objects with interactivity opens new avenues for exploration in clothing-based input sensors and the design of compliant mechanisms and other soft functional parts. More broadly, our work allows HCI practitioners to combine the aesthetics and flexibility of textiles with the rigidity and functionality of 3D prints. 


\section{ACKNOWLEDGMENTS}

We wish to thank Peregrine Hawthorn and Michael Longley at the Rochester Institute of Technology for their valuable fabrication assistance. This work was funded in part by the National Science Foundation under grant NSF IIS-1464377.

This material is based upon work funded and supported by the Department of Defense under Contract No. FA8721-05C-0003 with Carnegie Mellon University for the operation of the Software Engineering Institute, a federally funded research and development center. [Distribution Statement A] This material has been approved for public release and unlimited distribution. Please see Copyright notice for non-US Government use and distribution. DM-0004343

\section{REFERENCES}

1. 3D Systems. 2015. How to create a fabricate 3D tech-style fashion. (2015).

https : //www . youtube . com/watch?v=qKiHso484vw

2. Lea Albaugh. 2015. Lea, making things: clothes that move: why, and how? (2015).

http: //www . instamatique. com/blog/clothes-that-movewhy-and-how/

3. All3DP. 2016. 27 great 3D printer filament types (a guide). (2016). https://all3dp. com/best-3d-printerfilament-types-pla-abs-pet-exotic-wood-metal/

4. David Baraff and Andrew Witkin. 1998. Large steps in cloth simulation. In Proceedings of the 25th annual conference on Computer graphics and interactive techniques - SIGGRAPH '98. ACM Press, New York, New York, USA, 43-54. DOI:

http://dx.doi . org/10.1145/280814.280821

5. E.J.W. Barber. 1991. Prehistoric textiles: The development of cloth in the neolithic and bronze ages with special reference to the aegean. Princeton University Press. https://books . google. com/books?id=HnSlynSfeEIC

6. Benjamin Bridgens and Matthew Birchall. 2012. Form and function: The significance of material properties in the design of tensile fabric structures. Engineering Structures 44 (2012), 1 - 12. DOI :

http: //dx . doi . org/10.1016/j . engstruct . 2012.05.044

7. Robert Bridson, Ronald Fedkiw, and John Anderson. 2002. Robust treatment of collisions, contact and friction for cloth animation. In Proceedings of the 29th annual conference on Computer graphics and interactive techniques - SIGGRAPH '02, Vol. 21. ACM Press, New York, New York, USA, 594. DOI :

http: //dx. doi . org/10.1145/566570. 566623

8. I-Ming Chen, Shusong Xing, R. Tay, and Song Huat Yeo. 2005. Many strings attached: from conventional to robotic marionette manipulation. IEEE Robotics Automation Magazine 12, 1 (Mar 2005), 59-74. DOI : http://dx. doi . org/10.1109/MRA. 2005.1411420

9. Stelian Coros, Bernhard Thomaszewski, Gioacchino Noris, Shinjiro Sueda, Moira Forberg, Robert W. Sumner, Wojciech Matusik, and Bernd Bickel. 2013. Computational Design of Mechanical Characters. ACM
Trans. Graph. 32, 4, Article 83 (Jul 2013), Article 83, 12 pages. DOI: http://dx. doi .org/10.1145/2461912.2461953

10. Erik D. Demaine and Martin L. Demaine. 2001. Recent results in computational origami. In Origami $^{3}$ :

Proceedings of the 3rd International Meeting of Origami Science, Math, and Education (OSME 2001). A K Peters, Monterey, California, 3-16.

11. Stephen Eichhorn, John W.S. Hearle, Mike Jaffe, and Takeshi Kikutani. 2009. Handbook of textile fibre structure: Natural, regenerated, inorganic and specialist fibres. Vol. 2. Elsevier.

12. Michael Eisenberg. 2003. Mindstuff educational technology beyond the computer. Convergence: The International Journal of Research into New Media Technologies 9, 2 (2003), 29-53.

13. John W.S. Hearle. 1967. The structural mechanics of fibers. In Journal of Polymer Science Part C: Polymer Symposia, Vol. 20. Wiley Online Library, 215-251.

14. John W.S. Hearle, Percy Grosberg, and Stanley Backer. 1969. Structural mechanics of fibers, yarns, and fabrics. Wiley-Interscience.

15. John W.S. Hearle and William E. Morton. 2008. Physical properties of textile fibres. Elsevier.

16. Felix Heibeck, Basheer Tome, Clark Della Silva, and Hiroshi Ishii. 2015. uniMorph: Fabricating thin film composites for shape-changing interfaces. In Proceedings of the 28th Annual ACM Symposium on User Interface Software \& Technology (UIST'15). ACM, New York, NY, USA, 233-242. DOI :

http: //dx. doi .org/10.1145/2807442.2807472

17. Scott E. Hudson. 2014. Printing Teddy Bears: A Technique for 3D Printing of Soft Interactive Objects. In Proceedings of the SIGCHI Conference on Human Factors in Computing Systems (CHI'14). ACM, New York, NY, USA, 459-468. DOI : http://dx. doi . org/10.1145/2556288.2557338

18. Instructables. 2016. How to 3D print onto fabric. (2016). http: //Www . instructables . com/id/How-to-3D-Print-OntoFabric/

19. Gierad Laput, Xiang 'Anthony' Chen, and Chris Harrison. 2015. 3D printed hair: fused deposition modeling of soft strands, fibers, and bristles. In Proceedings of the 28th Annual ACM Symposium on User Interface Software \& Technology (UIST'15). ACM, New York, NY, USA, 593-597. DOI :

http://dx. doi .org/10.1145/2807442.2807484

20. R. Melnikova, A. Ehrmann, and K. Finsterbusch. 2014. 3D printing of textile-based structures by fused deposition modelling (FDM) with different polymer materials. In IOP Conference Series: Materials Science and Engineering, Vol. 62. IOP Publishing, 012018.

21. Jussi Mikkonen, Reetta Myllymäki, Sari Kivioja, Santeri Vanhakartano, and Helena Suonsilta. 2013. Printed material and fabric. Nordes 1, 5 (2013). 
22. Stefanie Mueller, Sangha Im, Serafima Gurevich, Alexander Teibrich, Lisa Pfisterer, François Guimbretière, and Patrick Baudisch. 2014a. WirePrint: 3D printed previews for fast prototyping. In Proceedings of the 27th annual ACM Symposium on User Interface Software \& Technology (UIST'14). ACM, 273-280.

23. Stefanie Mueller, Tobias Mohr, Kerstin Guenther, Johannes Frohnhofen, Kai-Adrian Rollmann, and Patrick Baudisch. 2014b. faBrickation: Fast 3D printing of functional objects by integrating construction kit building blocks. In CHI '14 Extended Abstracts on Human Factors in Computing Systems (CHI EA '14). ACM, New York, NY, USA, 527-530. DOI :

http://dx. doi .org/10.1145/2559206.2574779

24. Grace Ngai, Stephen C.F. Chan, Joey C.Y. Cheung, and Winnie W.Y. Lau. 2009. The TeeBoard: An Education-friendly Construction Platform for e-Textiles and Wearable Computing. In Proceedings of the SIGCHI Conference on Human Factors in Computing Systems (CHI '09). ACM, New York, NY, USA, 249-258. DOI : http://dx. doi .org/10.1145/1518701.1518742

25. Simon Olberding, Sergio Soto Ortega, Klaus Hildebrandt, and Jürgen Steimle. 2015. Foldio: Digital fabrication of interactive and shape-changing objects with foldable printed electronics. In Proceedings of the 28th Annual ACM Symposium on User Interface Software \& Technology (UIST'15). ACM, New York, NY, USA, 223-232. DOI :

http://dx. doi . org/10.1145/2807442 . 2807494

26. Eujin Pei, Jinsong Shen, and Jennifer Watling. 2015. Direct 3D printing of polymers onto textiles: experimental studies and applications. Rapid Prototyping Journal 21, 5 (Aug 2015), 556-571. DOI : http://dx . doi . org/10.1108/RPJ-09-2014-0126

27. Huaishu Peng, Jennifer Mankoff, Scott E. Hudson, and James McCann. 2015. A layered fabric 3D printer for soft interactive objects. In Proceedings of the 33rd Annual ACM Conference on Human Factors in Computing Systems (CHI'15), Vol. 1. ACM Press, New York, New York, USA, 1789-1798. DOI :

http://dx. doi.org/10.1145/2702123.2702327

28. Hannah Perner-Wilson, Leah Buechley, and Mika Satomi. 2011. Handcrafting Textile Interfaces from a Kit-of-no-parts. In Proceedings of the Fifth International Conference on Tangible, Embedded, and Embodied Interaction (TEI '11). ACM, New York, NY, USA, 61-68. DOI : http://dx.doi .org/10.1145/1935701.1935715

29. Ivan Poupyrev, Nan-Wei Gong, Shiho Fukuhara, Mustafa Emre Karagozler, Carsten Schwesig, and
Karen E. Robinson. 2016. Project Jacquard: Interactive digital textiles at scale. In Proceedings of the $2016 \mathrm{CHI}$ Conference on Human Factors in Computing Systems (CHI '16). ACM, New York, NY, USA, 4216-4227. DOI : http://dx. doi . org/10.1145/2858036.2858176

30. Daniela K. Rosner and Kimiko Ryokai. 2008. Spyn: Augmenting knitting to support storytelling and reflection. In Proceedings of the 10th International Conference on Ubiquitous Computing (UbiComp '08). ACM, New York, NY, USA, 340-349. DOI : http://dx.doi.org/10.1145/1409635.1409682

31. Lilia Sabantina, Franziska Kinzel, Andrea Ehrmann, and Karin Finsterbusch. 2015. Combining 3D printed forms with textile structures-mechanical and geometrical properties of multi-material systems. In IOP Conference Series: Materials Science and Engineering, Vol. 87. IOP Publishing, 012005.

32. Ashish Kumar Sen. 2007. Coated textiles: principles and applications. Crc Press.

33. Michael J. Sinclair and Kerwin Wang. 2003. Thermal actuator improvements: tapering and folding. In Proceedings of SPIE - The International Society for Optical Engineering, Jung-Chih Chiao, Vijay K. Varadan, and Carles CanÂL' (Eds.). 237-251. DOI :

http: //dx. doi .org/10.1117/12.499129

34. Alexander Teibrich, Stefanie Mueller, François Guimbretière, Robert Kovacs, Stefan Neubert, and Patrick Baudisch. 2015. Patching physical objects. In Proceedings of the 28th Annual ACM Symposium on User Interface Software \& Technology (UIST'15). ACM, New York, NY, USA, 83-91. D0I :

http://dx. doi . org/10.1145/2807442.2807467

35. Cesar Torres, Tim Campbell, Neil Kumar, and Eric Paulos. 2015. HapticPrint: Designing feel aesthetics for 3D printing. In Proceedings of the 28th Annual ACM Symposium on User Interface Software \& Technology UIST '15. ACM Press, New York, New York, USA, 583-591. DOI :

http://dx. doi .org/10.1145/2807442.2807492

36. John T. Williams. 2009. Textiles for cold weather apparel. Elsevier.

37. Lining Yao, Ryuma Niiyama, Jifei Ou, Sean Follmer, Clark Della Silva, and Hiroshi Ishii. 2013. PneUI: Pneumatically actuated soft composite materials for shape changing interfaces. In Proceedings of the 26th annual ACM Symposium on User Interface Software \& Technology (UIST'13). ACM, ACM, 13-22. 